\title{
Matrix metalloproteinases in Vietnamese patients with colorectal cancer
}

\author{
THAO PHUONG BUI ${ }^{1}$, ANH NGOC HOANG ${ }^{1}$, PHUONG LAN LE ${ }^{1}$, BICH THI PHAM ${ }^{1}$, \\ LINH THI TU NGUYEN ${ }^{1}$, HA MINH DO ${ }^{1}$, TO VAN TA ${ }^{2}$ and THAI HONG TRINH ${ }^{1}$ \\ ${ }^{1}$ Department of Biology and Key Laboratory of Enzyme and Protein Technology, College of Science, \\ Vietnam National University; ${ }^{2}$ Department of Anatomical Pathology-Cytopathology, \\ Vietnam National Cancer Hospital, Hanoi 10000, Vietnam
}

Received August 3, 2015; Accepted November 30, 2016

DOI: $10.3892 / 01.2017 .5680$

\begin{abstract}
Matrix metalloproteinases (MMPs), a family of endopeptidases also known as gelatinases, have been reported to affect the acquisition of the cell proliferative, cell invasive and metastatic phenotype of several types of cancer. In particular, the gelatinases MMP-2 and -9 have been revealed to facilitate tumor growth and invasion in patients with colorectal cancer (CRC). However, it is not known whether the gelatinase activity of MMP-2 and -9 is also elevated in Vietnamese patients with CRC. The activity of MMP-2 and -9 in the tissue samples of 103 patients with CRC was evaluated by gelatin zymography and quantified using ImageJ. The association between the level of activity of MMP-2 and -9 and various clinicopathological factors was analyzed, and Chisio BioPAX Editor software was used to visualize the biological pathways regulating the activity of the MMPs. The present study noticed significantly increased activity of active MMP-2 and MMP-9 in tumor tissues $(\mathrm{P}<0.01)$, and significantly decreased levels of pro-form MMP-2 and MMP-9 in tumor tissues $(\mathrm{P}<0.01)$, compared with that in adjacent tissues in patients with CRC. A correlation between the normalized different activity of MMP-2 and -9 and various clinicopathological features was observed. Furthermore, bioinformatics analysis indicated that the alteration in the activity of MMP-2 and MMP-9 may have been controlled by biological pathways involving the tissue inhibitors of metalloprotease- 2 and -1 . These findings indicate that the activity of the gelatinases MMP-2 and -9 affects the tumor progression and metastasis of patients with $\mathrm{CRC}$, providing a potential novel approach for determining the prognosis of CRC.
\end{abstract}

Correspondence to: Dr Thai Hong Trinh, Department of Biology and Key Laboratory of Enzyme and Protein Technology, College of Science, Vietnam National University, 334 Nguyen Trai, Thanh Xuan, Hanoi 10000, Vietnam

E-mail: thaith@vnu.edu.vn

Key words: colorectal cancer, gelatin zymography, MMP-2, MMP-9

\section{Introduction}

Colorectal cancer (CRC) is the third most common type of cancer worldwide (1), ranking fourth with respect to lethality level with 694,000 mortalities in 2012 (2). Early diagnosis by population-wide screening and identification of novel molecular markers may be a solution to decrease the mortality rate of $\mathrm{CRC}$.

The incidence of CRC is hypothesized to be lower in Asian countries than in the Western continent (3). However, the incidence of CRC has gradually grown over previous years due to the acquisition of a Western lifestyle, resulting in the increased consumption of red meat and alcohol in Asia (4). In Vietnam, the incidence of CRC is only exceeded by lung, liver and stomach cancer for men, and ranks fifth with respect to cancer incidence for women (5). However, national guidelines on CRC screening for Vietnamese patients remain poor.

Matrix metalloproteinases (MMPs) or gelatinases, a group of zinc-dependent endopeptidases involved in the degradation of the extracellular matrix (ECM), serve a critical role in the regulation of cell growth and other physiological processes (6). MMPs are also demonstrated to serve a role in tumor initiation, invasion, angiogenesis and metastasis by breaking down the connections between cells and between the cells and the matrix, which function as tissue barriers $(7,8)$. Numerous studies indicate that MMP-9 and MMP-2 may act as prospective biomarkers for CRC (9-11). In addition, MMP-9 and MMP-2 have been suggested to contribute to the inflammatory process in inflammatory bowel diseases (12). However, the role of gelatinase activity in Vietnamese patients with CRC remains unknown, although a preliminary study has been performed (13).

The present study used gelatin zymography to detect the levels of activity of MMP-2 and MMP-9, and established the association between their activity and various clinicopathological features in a cohort of Vietnamese patients with CRC. The results demonstrate that the activity levels of active MMP-2 and MMP-9 in tumor tissue increased compared with those in adjacent tissue. By contrast, the activity of pro-form MMP-2 and MMP-9 was noticed to be lower in tumor tissue compared with that in adjacent non-tumorous tissue. Since changes in expression and activity usually result from genomic alterations 
via a complicated interplay between multiple genes and their products, the present study identified certain potential biological pathways that may regulate the expression of MMPs. The analysis revealed the critical role of tissue inhibitors of metalloproteinases (TIMPs) in the control of MMP activity in cells and CRC. The results of the present study suggest that MMP-2 and MMP-9 may be useful for the prognosis of Vietnamese patients with CRC.

\section{Materials and methods}

Materials. A total of 103 pairs of tissue samples of tumors and adjacent tissues, $\sim 5-10 \mathrm{~cm}$ from the tumors of patients with CRC were obtained immediately upon surgical resection between August 2012 to June 2014 and stored at $-80^{\circ} \mathrm{C}$ until required. These samples were provided by the Department of Anatomical Pathology-Cytopathology, Vietnam National Cancer Hospital (Hanoi, Vietnam), who also classified the patients with cancer according to the tumor node metastasis classification (14). The study was approved by the local Ethics Committee of Vietnam National University (Hanoi, Vietnam) and informed consent was obtained from all patients. The standard enzymes used in the present study proform (pro)MMP-2, proMMP-9, active MMP-2 and active MMP-9, were obtained from Calbiochem (EMD Millipore, Billerica, MA, USA).

Sample preparation. The tissue samples were manually homogenised in $50 \mathrm{mM}$ Tris- $\mathrm{HCl}$ (pH 7.5), $75 \mathrm{mM} \mathrm{NaCl}, 1 \%$ [volume/volume (v/v)] Triton X-100 and 0.1\% [weight/volume (w/v)] SDS, and centrifuged at $4,500 \times \mathrm{g}$ for $20 \mathrm{~min}$ at $4^{\circ} \mathrm{C}$. The protein content of the resulting tissue extracts was determined by the Bradford method using bovine serum albumin (Sigma-Aldrich; Merck Millipore, Darmstadt, Germany) as a standard.

Gelatin zymography. Gelatin zymography was performed to quantify the activity of the activated and proenzyme forms of the gelatinases MMP-2 and MMP-9 in the tissue extracts, as described by Murnane et al (15). Each extract was diluted in the appropriate ratio with loading buffer $[0.125 \mathrm{M}$ Tris-HCl (pH 6.8), 17.4\% (v/v) glycerol, 4\% (w/v) SDS and $0.01 \%(\mathrm{w} / \mathrm{v})$ bromophenol blue], and loaded on a $10 \%(\mathrm{w} / \mathrm{v})$ SDS-polyacrylamide gel containing $0.1 \%(\mathrm{w} / \mathrm{v})$ gelatin (Sigma-Aldrich; Merck Millipore) as a substrate.

Subsequent to running, the gels were washed 3 times in $2.5 \%$ (v/v) Triton X-100 for $30 \mathrm{~min}$ at room temperature to remove the SDS. The gels were incubated overnight at $37^{\circ} \mathrm{C}$ in $50 \mathrm{mM}$ Tris- $\mathrm{HCl}$ (pH 8.0), $150 \mathrm{mM} \mathrm{NaCl}$ and $10 \mathrm{mM} \mathrm{CaCl}_{2}$ under gentle agitation to recover the activity of the enzymes. The gels were stained for 45 min with $0.25 \%$ (w/v) Coomassie Brilliant Blue R250 dissolved in $40 \%$ (v/v) methanol and $10 \%$ (v/v) glacial acetic acid. Proteolytic activities were visualised as clear zones against a dark blue background, indicating lysis of gelatin.

A total of $0.5 \mathrm{ng}$ per lane of recombinant proMMP-2, active MMP-2, proMMP-9 and active MMP-9 were loaded as standards to identify the bands corresponding to each enzyme and to allow the comparison of their activities (Fig. 1). The activation of recombinant proMMP-2 and MMP-9 was achieved by incubation with $1 \mathrm{mM}$ p-amino-phenylmercuric acetate (APMA), as recommended by the manufacturer (Sigma-Aldrich; Merck Millipore).

Quantification of the proteinase activity using ImageJ. ImageJ software (version 1.48; National Institutes of Health, Bethesda, MD, USA) was used to compare the expression levels of MMPs between the samples. To normalize the data between the lanes and the samples, the pixel density of each band in a gel was divided by the total pixel density of all bands in the same lane to yield the 'normalized value'. Normalized values of each band are calculated using the following formula: $\mathrm{X}(\%)=\left(100 \mathrm{xS}_{\mathrm{n}}\right) / \sum \mathrm{S}_{\mathrm{i}}$, where $\mathrm{S}_{\mathrm{n}}$ is the area value of the $\mathrm{nth}$ band in a lane; $\sum \mathrm{S}_{\mathrm{i}}$ is the total of area value of all the bands in a lane; and $\mathrm{X}$ is the normalized intensity value.

Visualization of pathways involving MMP-2 and MMP-9 using Chisio BioPAX Editor (ChiBE). ChiBE is a free software tool designed to visualize and analyze human pathways through the combination of deep pathway information, diverse genomic data and expression profiles (16). In the present study, ChiBE (version 2.2.0; https://code.google.com/p/chibe) was used to globally represent the pathway connections associated with MMP-2 and MMP-9, which were input as queries.

Statistical analysis. Comparisons between MMP levels in tumor tissue and adjacent tissue were performed using statistical tests, including Student's $t$-test and Kruskal-Wallis test. $\mathrm{P}<0.05$ was considered to indicate a as statistically significant difference.

\section{Results}

Activity of MMPs by gelatin zymography. The present study aimed to determine the precise location of active and pro-forms of MMPs in the gel. Fig. 1 illustrates the presence and location of active MMPs and proMMPs derived from standard enzymes and tissue extracts of two patients with CRC, patients no. 39002 and 42527, which were representative of all the cases evaluated. Zymography clearly identified the location of standard active MMP-2 (lane active MMP-2; Fig. 1A), two bands of standard active MMP-9 (lane active MMP-9; Fig. 1A), two bands corresponding to standard proMMP-2 (lane proMMP-2, Fig. 1A) and a band representing standard proMMP-9 (lane proMMP-9, Fig. 1A).

In the tumor sample, the location of the bands corresponding to proMMP-2 and active MMP-2 (Fig. 1B) was identified from the bands in the standards (Fig. 1A). These data also revealed the inverse activity of the bands corresponding to proMMP-2 and active MMP-2 observed in the two patients (Fig. 1B). Thus, subsequent to being activated, the area of the band corresponding to active MMP-2 increased by 1.8 -fold, while that of proMMP- 2 decreased by 1.8 -fold (Fig. 1B, case no. 39002). The effect of activation was revealed in a tissue sample from patient no. 42527, where treatment with APMA increased the activity of the band corresponding to active MMP-2 by 18 -fold (Fig. 1B).

Based on the aforementioned defined locations of the active and proforms of MMP-2 and MMP-9, the present study carried out gelatin zymography for 103 pairs of samples from patients with CRC. The analysis identified the presence of proMMP-9, 

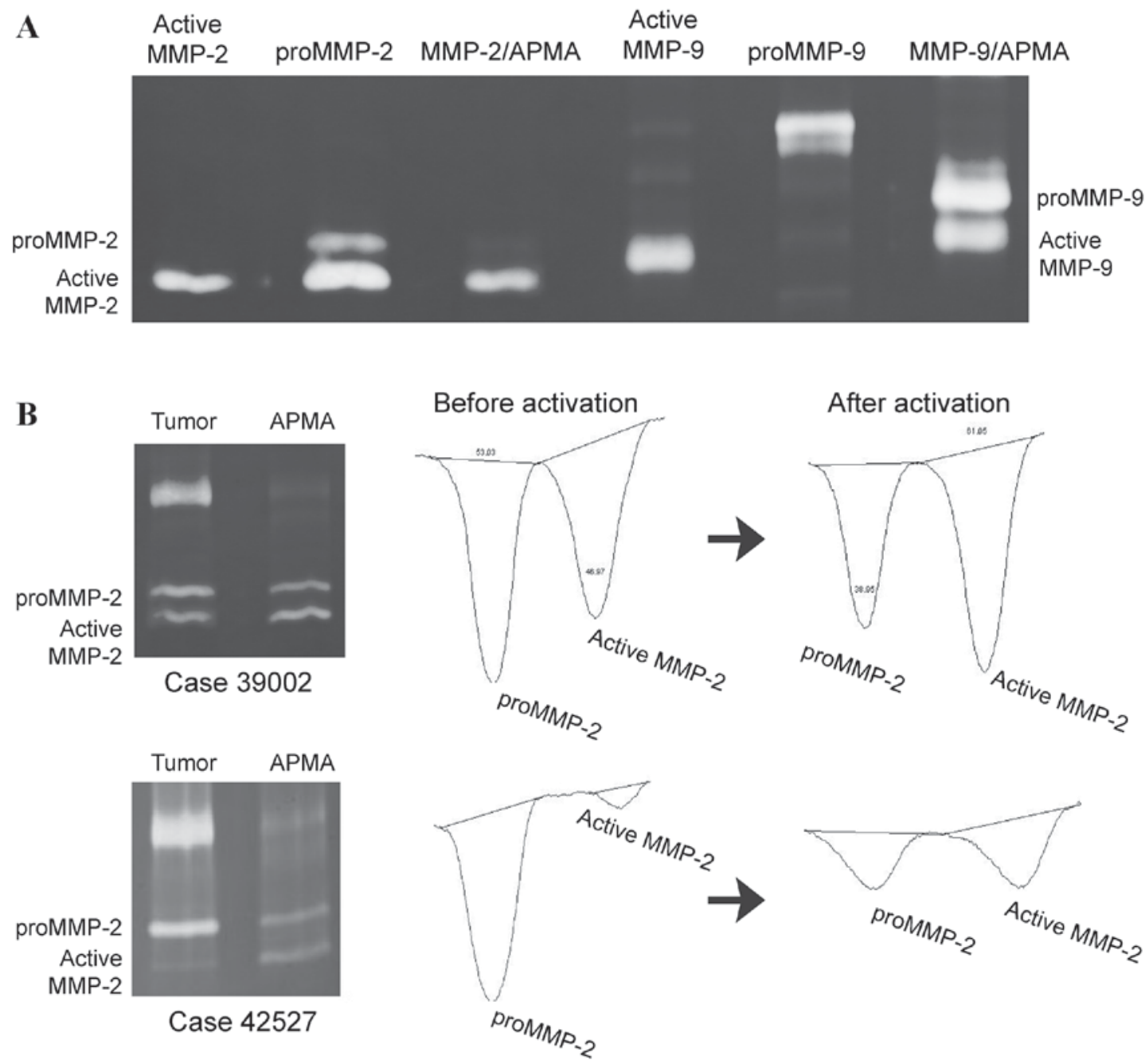

Figure 1. Gelatinase activity of MMPs in human colorectal cancer tissue extracts detected by quantitative gelatin zymography. (A) The position of proMMP-2, MMP-2, proMMP-9 and MMP-9 was confirmed using standard enzymes and APMA, ratio 10:1. (B) Gelatinase activity of active and proforms of MMP-2 in tumor samples from patients no. 39002 and 42527 prior and subsequent to activation by APMA. MMP-2 and proMMP-2 activities were quantified using ImageJ. MMP, matrix metalloproteinase; pro, proform; APMA, p-amino-phenylmercuric acetate.

active MMP-9, proMMP-2 and active MMP-2 in the tissue samples of Vietnamese patients with CRC (Fig. 2). Overall, in the majority of the samples, the activity of active MMP-2 and MMP-9 increased in the tumor tissues compared with that in the adjacent tissues. These results were additionally used to measure the levels of the active and proforms of MMP-2 and MMP-9 for each sample using ImageJ. In addition, the present study observed the appearance of other bands of larger molecular weight (Fig. 2), which may be due to the tissue extracts containing other types of proteinases exhibiting gelatinase activity.

The levels of active MMP-2 and MMP-9 in tumor tissues were revealed to be significantly higher than those in adjacent tissues. The normalized activity of active MMP-2 and MMP-9 was $\sim 1.9$ times higher in the tumor than in the adjacent tissue $(\mathrm{P}<0.001$; Table I). The method used in the present study also allowed the calculation of the active/proenzyme ratio in the two types of sample. The ratio of active MMP-2/proMMP-2 and active MMP-9/proMMP-9 of tumor tissue was double that of adjacent tissue $(\mathrm{P}<0.001$; Table I). This may associated with the decrease in the level of proMMP-2 and proMMP-9 in tumor tissue in comparison with that in adjacent tissue $(\mathrm{P}<0.01$; Table I).
Association between the activity of active MMPs, proMMPs and clinicopathological parameters of patients with CRC. Table II presents the association between the activities of active MMP-2 and MMP-9, their proforms and several clinicopathological features of patients with CRC. The normalized different activity was defined as the difference between the level of activity of a certain enzyme in the tumor and that in the adjacent tissue in each group. The normalized different activity of proMMP-2 exhibited a significant positive correlation with the extent of lymph node metastasis ( $\mathrm{N}$ stage; $\mathrm{P}=0.049$ ) and the extent of tumor invasion ( $\mathrm{T}$ stage; $\mathrm{P}=0.030$ ). The level of activity of proMMP-2 in tumor tissue at T1 and $\mathrm{T} 2$ increased almost by 4 -fold compared with that at T3 and T4. The normalized different activity of active MMP-2 was inversely correlated with the size of the tumors $(\mathrm{P}=0.044$; Table I). In addition, no association was detected between age, gender or differentiation and MMP-2 activity ( $\mathrm{P}>0.05)$.

Table II demonstrates that the normalized different activity of proMMP-9 was different between genders $(\mathrm{P}=0.012)$. In the female group, the value was $1 / 3$ higher compared with that in the male group. Similarly, the present study observed an association between the level of active MMP-9 and T stage 
Table I. Normalized activity of MMP-2 and MMP-9 in tumor tissue and adjacent tissue of patients with colorectal cancer, $\mathrm{n}=103$.

\begin{tabular}{llcr}
\hline & \multicolumn{2}{c}{ Mean normalized activity (standard deviation) } \\
\cline { 2 - 4 } & Adjacent tissue & Tumor tissue & P-value \\
\hline MMP-2 & $23.9(19.5)$ & $18.6(11.1)$ & 0.007 \\
ProMMP-2 & $4.9(4.3)$ & $9.3(5.8)$ & $4.8 \times 10^{-9}$ \\
Active MMP-2 & 0.31 & 0.68 & $8.2 \times 10^{-8}$ \\
Ratio of active MMP-2/proMMP-2 & & $36.8(13.6)$ & 0.006 \\
MMP-9 & $42.3(16.9)$ & $11.7(8.9)$ & $2.9 \times 10^{-6}$ \\
ProMMP-9 & $6.1(8.6)$ & 0.38 & $2.0 \times 10^{-5}$ \\
Active MMP-9 & 0.19 & & \\
Ratio of active MMP-9/proMMP-9 & & & \\
\hline
\end{tabular}

MMP, matrix metalloproteinase; pro, proform.

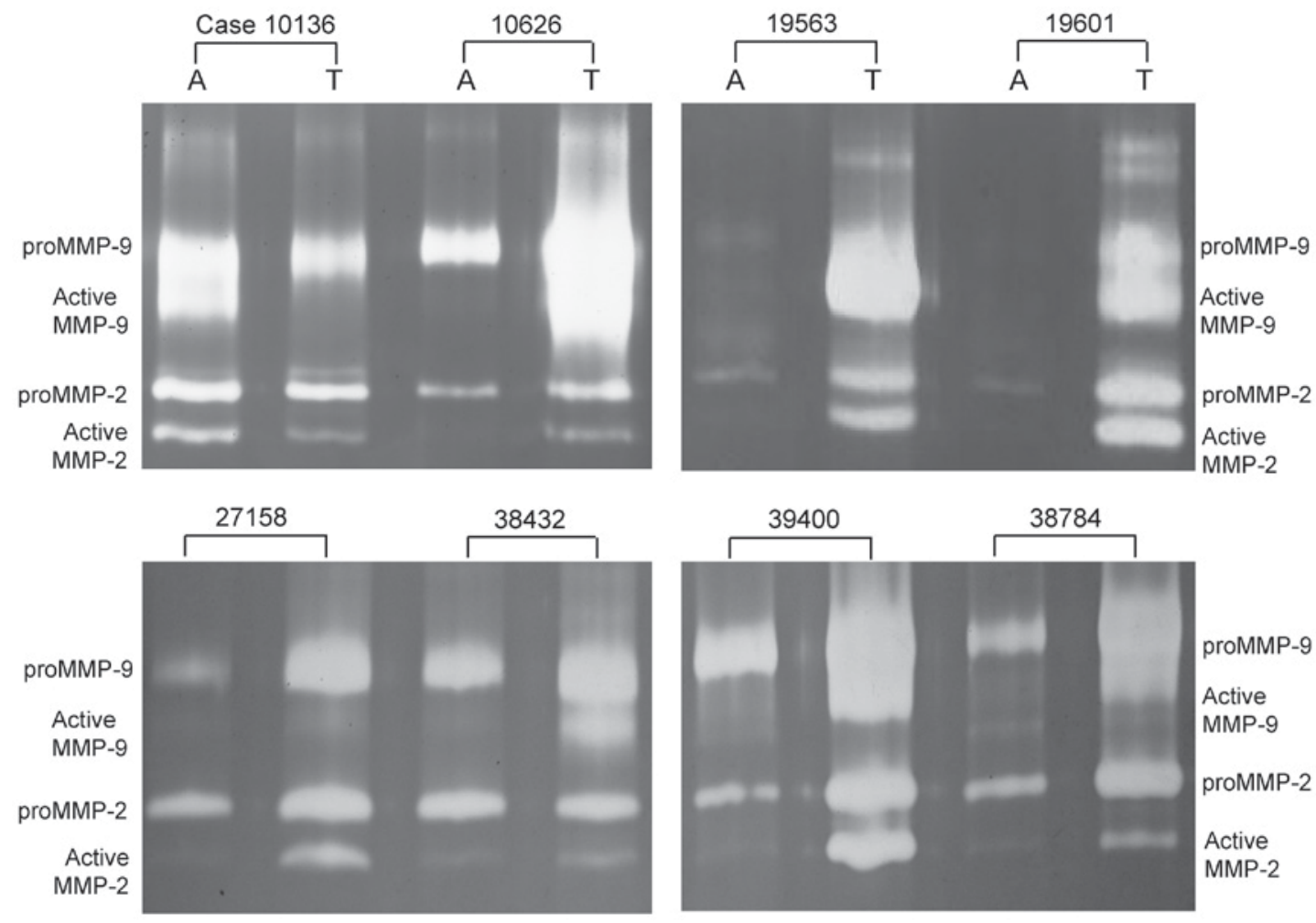

Figure 2. Gelatinase activity of the active and proforms of MMP-2 and MMP-9 in tissue samples of patients with colorectal cancer by quantitative gelatin zymography. Representative samples from patients no. 10136, 10626, 19563, 19601,27158,38432,39400 and 38784 are shown. MMP, matrix metalloproteinases; proMMP, pro-form MMP; A, adjacent tissue; T, tumor tissue.

$(\mathrm{P}=0.023)$, with tumor tissue at $\mathrm{T}$ stages 1 and 2 showing an almost 2-times higher level of normalized different activity compared with that of tumor tissue at T stages 3 and 4 . None of the remaining parameters exhibited a correlation with MMP-9 activity $(\mathrm{P}>0.05)$.

Biological pathways associated with MMP-2, MMP-9 and their changes in CRC. The present study then investigated the possible consequences of the alteration in the levels of activity of MMP-2 and MMP-9 in tumor tissue by evaluating the molecular connections of these MMPs to cellular pathway networks. The majority of MMPs exhibited close functional associations at the molecular level (Fig. 3). For example, MMP-2, MMP-7, MMP-9 and cluster of differentiation 44 form a complex that has a functional interaction with MMP-1. The $\alpha 2$-macroglobulin (A2M) complex consists of A2M and various MMPs, including MMP-1, MMP-3 and MMP-2. In addition, this network identified a catalytic connection between MMP-1 and MMP-9, suggesting that MMP-1 affects the activation of MMP-9. Notably, the present study detected the natural inhibitors of MMPs, TIMP-2 and TIMP-1, in the pathway landscape of MMP-2 and MMP-9, respectively. TIMP-2, MMP-14 and MMP-2 form a functional complex, similar to MMP-9 and TIMP-1. 
Table II. Normalized different activity of MMP-2 and MMP-9 in colorectal tissues vs. adjacent tissues and its association with clinicopathological parameters.

\begin{tabular}{|c|c|c|c|c|c|}
\hline \multirow[b]{2}{*}{ Parameter } & \multirow[b]{2}{*}{ No. of cases } & \multicolumn{4}{|c|}{ Normalized different activity } \\
\hline & & ProMMP-2 & Active MMP-2 & ProMMP-9 & Active MMP-9 \\
\hline \multicolumn{6}{|l|}{ Age, years } \\
\hline$<50$ & 22 & -9.8 & 3.8 & -5.1 & 9.4 \\
\hline$\geq 50$ & 81 & -4.7 & 4.4 & -5.2 & 5.5 \\
\hline P-value & & 0.180 & 0.361 & 0.298 & 0.086 \\
\hline \multicolumn{6}{|l|}{ Gender } \\
\hline Male & 42 & -7.7 & 5.4 & -4.1 & 7.9 \\
\hline Female & 61 & -4.4 & 3.4 & -5.8 & 5.3 \\
\hline P-value & & 0.089 & 0.218 & 0.012 & 0.132 \\
\hline \multicolumn{6}{|c|}{ Differentiation } \\
\hline High & 11 & 6.4 & 5.8 & -6.4 & 8.5 \\
\hline Moderate & 67 & -6.8 & 4.1 & -5.2 & 6.1 \\
\hline Poor & 6 & -5.8 & 1.9 & -4.7 & 11.9 \\
\hline P-value & & 0.174 & 0.533 & 0.967 & 0.356 \\
\hline \multicolumn{6}{|c|}{ Tumor size, cm } \\
\hline$<3.0$ & 27 & -9.5 & 6.1 & -3.4 & 8.9 \\
\hline $3.0-3.5$ & 36 & -3.1 & 5.2 & -7.5 & 4.6 \\
\hline$>3.5$ & 38 & -5.7 & 1.9 & -4.7 & 6.5 \\
\hline P-value & & 0.492 & 0.044 & 0.596 & 0.297 \\
\hline \multicolumn{6}{|l|}{$\mathrm{N}$ stage } \\
\hline N0 & 68 & -8.3 & 3.8 & -4.0 & 7.1 \\
\hline $\mathrm{N} 1,2$ & 35 & -7.9 & 5.1 & -7.4 & 4.8 \\
\hline P-value & & 0.049 & 0.201 & 0.186 & 0.159 \\
\hline \multicolumn{6}{|l|}{ T stage } \\
\hline $\mathrm{T} 1,2$ & 35 & -11.8 & 5.0 & -5.0 & 9.3 \\
\hline $\mathrm{T} 3,4$ & 68 & -2.6 & 3.9 & -5.2 & 4.8 \\
\hline P-value & & 0.030 & 0.243 & 0.476 & 0.023 \\
\hline
\end{tabular}

MMP, matrix metalloproteinase; pro, proform.

\section{Discussion}

The present study demonstrated that active MMP-2 and MMP-9 levels are significantly increased in tumor tissue compared with those in adjacent tissue of Vietnamese patients with CRC. This result is consistent with previous studies investigating the association between the activity of MMP-2 and MMP-9 and the progression of CRC $(15,17)$ Murnane et al (15) observed the expression of active MMP-2 in $99 \%$ of 269 patients with CRC, and $95 \%$ of these patients exhibited an elevated activity of active MMP-2 in tumor tissues, which was $\sim 10$ times higher than that in normal tissues. While the activity of proMMP-2 and proMMP-9 has also been shown to rise in tumor tissue compared with that in normal tissue $(15,17)$, the results of the present study demonstrate a lower level of activity of proMMP-2 and proMMP-9 in tumor samples compared with that in adjacent tissue, suggesting that the activation of MMP-2 and MMP-9 may be a crucial step in the process of tumor invasion. Indeed, the MMP-2 test was revealed to be the most effective with respect to the detection of CRC, with a sensitivity and specificity of 84 and 93\%, respectively (15).

By gelatin zymography, MMP activity has been verified in the breast tissues of Indian patients (18). Active MMP-2 and MMP-9 levels in malignant breast tissue were higher compared with that in adjacent tissue, and proMMP-2 levels were significantly higher in adjacent tissue compared with malignant breast tissue, which is consistent with the results of the present study. Weng et al (19) investigated the role of the activity of MMP-2 and MMP-9 in Chinese patients with early-stage lung cancer, revealing that MMP-9 activity decreased, while MMP-2 activity increased, in cancer tissues compared with that in normal samples (19). In addition, in Italian patients with bladder tumors, prostate cancer and renal carcinoma, the majority of cancerous urine samples contained MMP-9 activity, but only a small number contained MMP-2 activity (20-22).

The increased activity of active MMP-2 and MMP-9 in tumor tissue may be due to the decreased level of TIMP-2 and TIMP-1; however, in the scope of the present study, the impact 


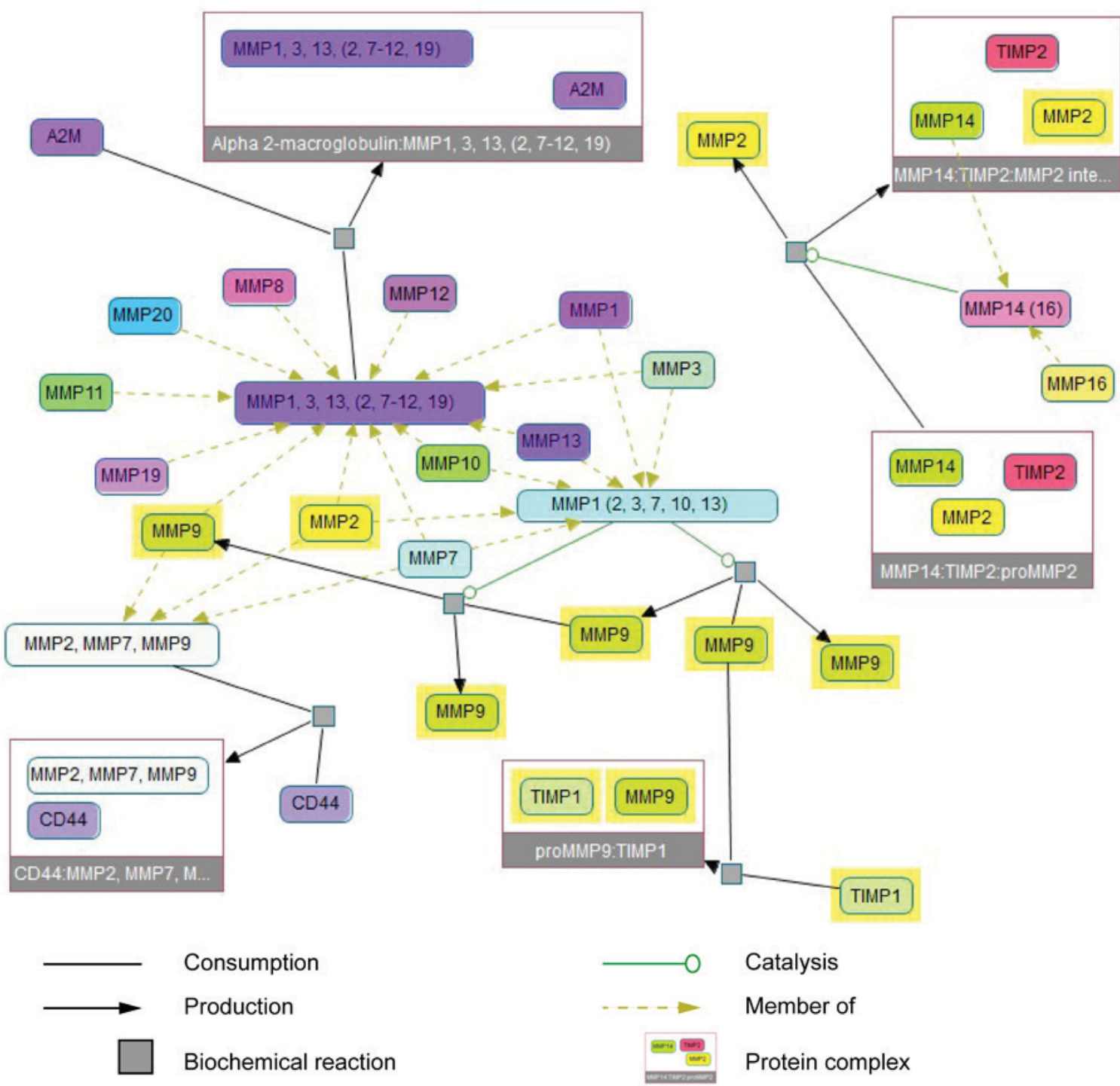

Figure 3. Network of biological pathways associated with MMP-2 and MMP-9. All molecular components interact with MMP-2 and MMP-9 by physical or functional interaction. A2M, $\alpha 2$-macroglobulin; CD, cluster of differentiation; MMP, matrix metalloproteinase; TIMP, tissue inhibitors of metalloproteinase.

of TIMPs was only considered with respect to the network. The association between TIMPs, MMPs and cancer remains unknown. Offenberg et al (23) revealed an elevated expression of TIMP-1 and a reduced expression of TIMP-2 in CRC samples in comparison with that in normal samples. In addition, an association between the downregulation of TIMP-2 expression and the increased activity of MMP-2 in patients with CRC patients has been identified $(24,25)$. By contrast, upregulation of TIMP-2 has been reported to promote MMP-2 activation and invasion in human glioblastoma cells (26). Utilizing bioinformatics tools, the present study explained the association between TIMP-2 and MMP-2 based on the biological pathways connecting them (Fig. 3). At the post-translational level, TIMP-2 forms a complex with MMP-14, which activates proMMP-2 on the tumor cell surface (27). From this point of view, there is a proportional correlation between the expression of TIMP-2 and MMP-2. Notably, the present study revealed that, besides MMP-14, MMP-16 may also contribute to catalysis through this type of interaction. In fact, MMP-2 has been observed to be activated by several members of the membrane-anchored MMP family, including MMP-14, MMP-15, MMP-16 and MMP-17 (27,28). The arrangement of the activated cell surface associated MMP-3 is well known, although cell surface-associated activator receptor mechanisms for MMP-9 remain unclear.

TIMP-1 is well known as the inhibitor of MMP-9, and the present study identified TIMP-1 in the pathway regulating the activity of MMP-9 in the network represented in Fig. 3. It has been shown that the expression of TIMP-1 increased in the tumor tissue of patients with CRC (29). Whether the impact of TIMP-1 on MMP-9 activity across different types of cancer is identical, however, remains unknown. In case of thymic epithelial tumors, neither MMP-9 nor TIMP-1 were observed to affect cancer invasiveness (30).

With respect to clinicopathological features, the present study revealed certain unexpected results. While the study of Waas et al (17) revealed no correlation between the expression or activity of active or proMMP-2 and MMP-9 and T stage, the results of the present study revealed a reverse correlation in the case of proMMP-2 and active MMP-9. Normalized different 
activity of proMMP-2 and active MMP-9 in tumors at T1 and T2 stages were 4 and 2-times higher than that of tumors at T3 and T4 stages, respectively (Table II). There was no correlation detected in the case of MMP-2 (Table II), which is consistent with the study of Waas et al (17). According to that study, the transition tissues located between the tumor and normal tissues of the patients with newly developing metastases express a higher level of enzyme activity than that exhibited by the group of patients without metastasis.

The present study observed that the normalized different activity of MMP-2 declined as the tumor size decreased. The degradation of the ECM structure by MMP-2 is required during the early stages of tumor development in order to destroy the surrounding connective tissues, and this is considered to be the consequence of the elevated expression of MMP-2 (31). However, when the tumor matures to a defined dimension, its invasion into the ECM may be reduced, which would result in a reduction in the activity of MMP-2, which was observed in the present study.

The present study identified a correlation between the level of MMP-9 activity and the invasive state of the tumor. Measuring protein expression in the tissue of patients with CRC, Kostova et al (32) noticed that the expression of MMP-9 exhibited a positive correlation with the level of tumor invasion. By contrast, no correlation was detected in the present study between active MMP-2 and the level of tumor invasion. The difference between MMP-2 and MMP-9 in terms of their association with $\mathrm{T}$ stage may be due to the diversification with respect to function. MMP-2 participates in a wide range of functional pathways, including remodeling of vasculature, tissue morphogenesis, regulating the bioavailability of several growth factors, inflammation (33) and atherosclerotic plaque rupture (34), whereas MMP-9 mainly serves a role in the local proteolysis of the ECM, angiogenesis and leukocyte migration (35).

In conclusion, the present study identified elevated levels of MMP-2 and MMP-9 activity in tumor tissues compared with those in adjacent tissues in Vietnamese patients with CRC. The present study clearly demonstrated the differences between MMP-2 and MMP-9 in terms of proteinase activity and correlation with clinical parameters. The present results suggest the importance of distinguishing the participation of active and proforms of MMPs in colorectal tumor progression. These results emphasize the potential application of MMPs in the development of CRC prognosis and the requirement of additional investigation into the mechanism of how changes in the levels of MMP expression elevate cancer, and whether differences between studies may be associated with differences in patient cohorts and ethnicity, which may affect disease onset and progression. Additional studies on a larger population of Vietnamese patients with CRC are required to define the importance of MMP-2 and MMP-9 as markers for $\mathrm{CRC}$. The data presented in the present study are prerequisite to a forthcoming study on the 5-year survival rate of patients with CRC.

\section{Acknowledgements}

The present authors would like to thank Dr Jan Dimberg (Jönköping University, Jönköping, Sweden) and Dr Dave
Fernig (University of Liverpool, Liverpool, UK) for their assistance. The present study was funded by grants from the Scientific and Technological Foundation of Vietnam National University (Hanoi, Vietnam; grant no. QGTĐ.13.06).

\section{References}

1. GLOBOCAN 2012: Cancer estimated incidence, mortality and prevalence worldwide in 2012. http://globocan.iarc.fr/old/factsheet.asp. Accessed May 6, 2015.

2. WHO: Cancer fact sheet N297. http://www.who.int/mediacentre/ factsheets/fs297/ en/. Accessed May 6, 2015.

3. Koo JH, Leong RW, Ching J, Yeoh KG, Wu DC, Murdani A, Cai Q, Chiu HM, Chong VH, Rerknimitr R, et al: Knowledge of, attitudes toward and barriers to participation of colorectal cancer screening tests in the Asia-Pacific region: A multicenter study. Gastrointest Endosc 76: 126-135, 2012.

4. Sung JJ, Lau JY, Goh KL and Leung WK; Asia Pacific Working Group on Colorectal Cancer: Increasing incidence of colorectal cancer in Asia: implications for screening. Lancet Oncol 6: 871-876, 2005.

5. Overview: National Strategy for Cancer Control (2010 and 2020). Viet Nam: 1-5, 2010.

6. Stöcker W, Grams F, Baumann U, Reinemer P, Gomis-Rüth FX, McKay DB and Bode W: The metzincins-topological and sequential relations between the astacins, adamalysins, serralysins, and matrixins (collagenases) define a superfamily of zinc-peptidases. Protein Sci 4: 823-840, 1995.

7. Pellikainen JM, Ropponen KM, Kataja VV, Kellokoski JK, Eskelinen MJ and Kosma VM: Expression of matrix metalloproteinase (MMP)-2 and MMP-9 in breast cancer with a special reference to activator protein-2, HER2, and prognosis. Clin Cancer Res 10: 7621-7628, 2004.

8. Iniesta P, Morán A, De Juan C, Gómez A, Hernando F, García-Aranda C, Frías C, Díaz-Lospez A, Rodríguez-Jiménez F, Balibrea JL and Benito M: Biological and clinical significance of MMP-2, MMP-9, TIMP-1 and TIMP-2 in non-small cell lung cancer. Oncol Rep 17: 217-223, 2007.

9. Liabakk NB, Talbot J, Wikinson K and Balkwill F: Matrix metalloprotease 2 (MMP-2) and matrix metalloprotease 9 (MMP-9) type IV collagenases in colorectal cancer. Cancer Res 56: 190-196, 1996.

10. Takeuchi T, Hisanaga M, Nagao M, Ikeda N, Fujii H, Koyama F, Mukowaga T, Matsumoto $\mathrm{H}$, Kondo S, Takahashi C, et al: The membrane-anchored matrix metalloproteinase (MMP) regulator RECK in combination with MMP-9 serves as an informative prognosis indicatior for colorectal cancer. Clin Cancer Res 10: 5572-5579, 2004.

11. Herszényi L, Hritz I, Lakatos G, Varga MZ and Tulassay Z: The behavior of matrix metalloproteinases and their inhibitors in colorectal cancer. Int J Mol Sci 13: 13240-13263, 2012.

12. Kurzeepa J, Madro A, Czechowska G, Kurzepa J, Celiński K, Kazmierak W and Słomka M: Role of MMP-2 and MMP-9 and their natural inhibitors in liver fibrosis, chronic pancreatitis and non-specific inflammatory bowel diseases. Hepatobiliary Pancreat Dis Int 13: 570-579, 2014.

13. Hoang NA, Nguyen VH, Bui PT, Le LP, Pham TB, Nguyen TTL, Do MH and Trinh HT: Activity of matrix metalloproteinases (MMP-2 and MMP-9) in colorectal cancer patients. Tap Chi Sinh Hoc. J Biol 37: 279-288, 2015.

14. Greene FL, Page DL, Fleming ID, Fritz AG, Balch CM, Haller DG and Morrow M, (eds): AJCC cancer staging manual. 6th edition. Lippincott Raven Publishers, Philadelphia, PA, pp113-123, 2002.

15. Murnane MJ, Cai J, Shuja S, McAneny D, Klepeis V and Willett JB: Active MMP-2 effectively identifies the presence of colorectal cancer. Int J Cancer 125: 2893-2902, 2009.

16. Babur Ö, Dogrusoz U, Çakir M, Aksoy BA, Schultz N, Sander C and Demir E: Integrating biological pathways and genomic profiles with ChiBE 2. BMC Genomics 15: 642, 2014.

17. Waas ET, Lomme RM, DeGroot J, Wobbes T and Hendriks T: Tissue levels of active matrix metalloproteinase-2 and -9 in colorectal cancer. Br J Cancer 86: 1876-1883, 2002.

18. Shah FD, Shukla SN, Shah PM, Shukla HK and Patel PS: Clinical significance of matrix metalloproteinase 2 and 9 in breast cancer. Indian J Cancer 46: 194-202, 2009.

19. Weng Y, Cai M, Zhu J, Geng J, Zhu K, Jin X and Ding W: Matrix metalloproteinase activity in early-stage lung cancer. Onkologie 36: 256-259, 2013. 
20. Di Carlo A, Terracciano D, Mariano A and Macchia V: Urinary gelatinase activities (matrix metalloproteinases 2 and 9) in human bladder tumors. Oncol Rep 15: 1321-1326, 2006.

21. Di Carlo A, Mariano A, Terracciano D, Ferro M, Montanaro V, Marsicano M, Di Lorenzo G, Altieri V and Macchia V: Matrix metalloproteinases-2 and -9 in the urine of prostate cancer patients. Oncol Rep 24: 3-8, 2010.

22. Di Carlo A: Matrix metalloproteinase-2 and -9 in the sera and in the urine of human oncocytoma and renal cell carcinoma. Oncol Rep 28: 1051-1056, 2012.

23. Offenberg $\mathrm{H}$, Brünner $\mathrm{N}$, Mansilla $\mathrm{F}$, Orntolt $\mathrm{TF}$ and Birkenkamp-Demtroder K: TIMP-1 expression in human colorectal cancer is associated with TGF-B1, LOXL2, INHBA1, TNF-AIP6 and TIMP-2 transcript profiles. Mol Oncol 2: 233-240, 2008

24. Kim TD, Song KS, Li G, Choi H, Park HD, Lim K, Hwang BD and Yoon WH: Activity and expression of urokinase-type plasminogen activator and matrix metalloproteinases in human colorectal cancer. BMC Cancer 6: 211, 2006.

25. Li BH, Zhao P, Liu SZ, Yu YM, Han M and Wen JK: Matrix metalloproteinase-2 and tissue inhibitor of metallo- proteinase-2 in colorectal carcinoma invasion and metastasis. World J Gastroenterol 11: 3046-3050, 2005.

26. Lu KV, Jong KA, Rajasekaran AK, Cloughesy TF and Mischel PS: Upregulation of tissue inhibitor of metalloproteinases (TIMP)-2 promotes matrix metalloproteinase (MMP)-2 activation and cell invasion in a human glioblastoma cell line. Lab Invest 84: 8-20 2004.

27. Nagase H, Visse R and Murphy G: Structure and function of matrix metalloproteinases and TIMPs. Cardiovasc Res 69 $562-573,2006$
28. Morrison CJ and Overall CM: TIMP independence of matrix metalloproteinase (MMP)-2 activation by membrane type 2 (MT2)-MMP is determined by contributions of both the MT2-MMP catalytic and hemopexin C domains. J Biol Chem 281: 26528-26539, 2006.

29. Gong Y, Scott E, Lu R, Xu Y, Oh WK and Yu Q: TIMP-1 promotes accumulation of cancer associated fibroblasts and cancer progression. PLoS One 8: e77366, 2013.

30. Sogawa K, Kondo K, Fujino H, Takahashi Y, Miyoshi T, Sakiyama S, Mukai K and Monden Y: Increased expression of matrix metalloproteinase-2 and tissue inhibitor of metalloproteinase- 2 is correlated with poor prognostic variables in patients with thymic epithelial tumors. Cancer 98: 1822-1829, 2003.

31. Campbell NE, Kellenberger L, Greenaway J, Moorehead RA, Linnerth-Petrik NM and Petrik J: Extracellular matrix proteins and tumor angiogenesis. J Oncol 2010: 586905, 2010.

32. Kostova E, Slaninka-Miceska M, Labacevski N, Jakovski K, Trojachanee J, Atanasovska E, Janevski V, Jovanovik R and Janevska V: Expression of matrix metalloproteinases 2, 7 and 9 in patients with colorectal cancer. Vojnosanit Pregl 71: 52-59, 2014.

33. Vu TH and Werb Z: Matrix metalloproteinases: Effectors of development and normal physiology. Genes Dev 14: 2123-2133, 2000.

34. Newby AC, George SJ, Ismail Y, Johnson JL, Sala-Newby GB and Thomas AC: Vulnerable atherosclerotic plaque metalloproteinases and foam cell phenotypes. Thromb Haemost 101: 1006-1011, 2009.

35. Barrick B, Campbell EJ and Owen CA: Leukocyte proteinase in wound healing: Roles in physiologic and pathologic processes. Wound Repair Regen 7: 410-422, 1999. 